\title{
Genioglossus Response to Airway Occlusion in Apneic Versus Nonapneic Infants
}

\author{
FSTFIII: B GAUDA MARTHA J MILIER. WAIDIMAR A. (ARI.O. JULIANN M. IDIFIORL: \\ DAVID (. JOHNSEN. ANI) RI(HARI) J. MARTIN \\ Departments of Pediatrics and Pediatric Dentistry at Rainhow Babies and Childrens Hespital. Case Hextern \\ Reserve Lniversils: (leveland. Ohio 44106
}

\begin{abstract}
ABSTRACI. The ability to maintain pharyngeal patency is compromised in infants who have apneic episodes associated with airway obstruction. Since the genioglossus (GG) muscle is thought to be important in maintaining pharyngeal patency, we measured the GG EMG with sublingual surface electrodes during unobstructed breathing and in response to end-expiratory airway occlusion. Studies were performed in nine premature infants with mixed and obstructive apnea and in eight nonapneic control infants. Phasic GG EMG was usually absent during normal tidal breathing in both groups of infants, however, GG activity typically appeared during airway occlusion. The response of the GG muscle during airway occlusion differed between control and apneic infants. During the first three occluded inspiratory efforts, control infants had $42 \pm 5,74$ \pm 5 , and $80 \pm 5 \%$ (mean $\pm \mathrm{SEM}$ ) of their occlusions associated with a GG EMG response, respectively. In contrast, apneic infants had significantly fewer $(13 \pm 4,38$ \pm 9 , and $52 \pm 9 \%$ ) occlusions associated with a GG EMG response. There was a delay in onset of the CG EMG when compared to the onset of the diaphragm EMG and initial negative esophageal pressure swing, but this delay decreased with each subsequent appearance of the $\mathrm{GG}$ EMG in both infant groups. Infants with mixed and obstructive apnea thus have decreased activation of their $G G$ in response to occlusion which may reflect their inability to recruit dilating muscles of the upper airway during spontaneous airway obstruction. (Pediatr Res 22: 683-687. 1987)
\end{abstract}

\section{Abbreviations}

G(i, genioglossus

DI $\Lambda$, diaphragmatic

Ti, inspiratory time

Upper airway obstruction is a frequent component of apnea in adults with sleep apnea syndrome. in micrognathic infants. and in infants with apnea of prematurity $(1-3)$. The site of airway obstruction is primarily pharyngeal (4) and thus the response of the major upper airway dilating muscle, the $G(i$. has been a subject of recent investigation. The $(i)$ response to airway occlusion, hypercarbia, and hypoxia has been measured in adults. animals, and micrognathic infants by recording F.MG activity with needle electrodes inserted into the muscle $(2,5-7)$. As this technique is unsuitable for the study of (if activity in healthy

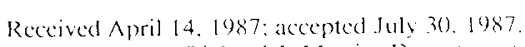

Correspondence Richard J. Martin, Department of Pediatries. Rainbow Babies and (hildrens Hospital. 2101 Adelhert Road. (leveland. ()H 44106.

Supported by VIll (irants 111.25830 and 151.31173. infants. we adapted the noninvasive sublingual surface electrode apparatus devised by Doble a al. (8) in adults to craluate the GG response in a group of premature infants.

To test the hypothesis that infants with mixed and obstructive apnea are less able to initiate an upper airway dilator response to airway occlusion. we used sublingual surface electrodes to examine the GG response to induced end-expiratory airway occlusion in infants with and without mixed and obstructive apnea. Our data indicate that GG activity does differ between infants with apnea and healthy control infants.

\section{SUBJFCTS AND MITHOIDS}

A study population of 17 premature infants was selected. Vine infants with mixed and obstructive apnea and eight nonapneic control infants were studied. Infants with apnea had a (mean \pm SD) gestational age of $28 \pm 2 \mathrm{wk}$ (range $26-31 \mathrm{wk}$ ). birth weight of $1165 \pm 290 \mathrm{~g}($ range $740-1675 \mathrm{~g})$, and a postconceptional age of $32 \pm 1 \mathrm{wk}$ (range 31-34wk). The control infants had a gestational age of $30 \pm 2 \mathrm{wk}$ (range 26-3.3 wk), birth weight of $1420 \pm 310$ (range $800-16.30 \mathrm{~g}$ ). and postconceptional age of 3.3 \pm 1 wk (range 31-35 wk). The two groups were prospectively selected to be of comparable postconceptional age and this was confirmed by parametric and nonparametric unpaired / test. Birth weight did not differ between the two groups. The two groups, however, did differ in gestational age at birth when compared by the Mann Whitner test $(p=0 .(14)$.

None of the infants required supplemental oxygen and there was no evidence of tachypnea or radiographic lung discase at time of the study. Infants with a history of three or more bradycardias with or without apnea, as detected by electrocardiogram and impedance monitoring. and occurring $24 \mathrm{~h}$ prior to the study period. were selected for the apneic group. Bradycardia without detection of apnea by impedance monitoring was assumed to be representative of obstructive apnea. Apncic infants also had two or more mixed and/or obstructive apneic cpisodes lasting at least 10 s during the 2-h study period. Vo comparable apneic or bradveardiac episodes were observed in the control inlants. Spontaneous apneic episodes were defined as: 1) obstructive if DIA EMG is and esophageal pressure swings persisted in the absence of nasal airflow for $\geq 10 s: 2$ ) mixed if in addition to the obstructed efforts there were eessation of nasal airflow, ahsent esophageal pressure swings. and absent diaphragmatic IMC $\mathrm{r}$ for at least $2 \mathrm{~s}$ with the entire episode lasting $\geq 1($ ) $s$. Apneas without any obstructive components (central) of $\geq 10$ s duration were not observed in either group of infants. Seven of nine infants with apnea and none of the control infants were receiving theophylline at the time of the study.

The infants were studied in the neonatal pulmonary rescarch laboratory in incubators maintained to approximate the infants' neutral thermal environment. Informed parental consent was obtained prior to each study. Measurements were performed 
during sleep, and no sedation was used. The apparatus used to record the GG EMG consisted of two silver, domed, 4-mm surface electrodes attached to flexible insulated wires and mounted on a moulded acrylic chin piece that was held in place by an elastic head band. The wires could be adjusted to allow a secure fit for each infant. The two surface electrodes were placed in an anterior sublingual position on each side of the frenulum (Fig. 1). The infants tolerated the apparatus well and were positioned with their head turned laterally and their neck in a neutral position. The DIA EMG was obtained from two adhesive surface electrodes (Medtronic Andover Medical, Lowell, MA) placed over the right subcostal margin between the mid-and anterior axillary line. The GG and DIA EMGs were amplified (Preamplifier, Coulbourn, Lehigh Valley, PA) and displayed on an oscilloscope. Raw EMGs were filtered from 30-300 Hz. For the DIA EMG, the electrocardiographic artifact was removed by gating (SB-1 EKG Blanker, CWE, Inc., Ardmore, PA). The raw DIA EMG was full-wave rectified and compared to a reference voltage that was adjusted to be triggered by the electrocardiogram. This signal was both stored undisturbed and sent to a delay circuit. The delayed signal was sent to the output until an electrocardiogram was detected. At this point, the delayed signal was replaced by the undelayed signal. Hence, the electrocardiogram was removed and replaced by an adjacent portion of the DIA EMG. This process introduced a delay of the DIA EMG of approximately $60 \mathrm{~ms}$. The delay was always accounted for when timing calculations were performed using the DIA EMG. Both EMGs were averaged by a moving time averager (9) (MA-821 moving averager, Charles Ward Enterprises, PA) with a time constant of $100 \mathrm{~ms}$. Esophageal pressure was measured by a dome transducer (Gould, Cleveland, $\mathrm{OH}$ ), attached to a $5 \mathrm{Fr}$ fluid-filled catheter (Argyle, St. Louis, MO) placed in the midesophagus. Airflow and mask pressure were measured with a nasal pneumotachograph that had a linear flow up to 5 liter $/ \mathrm{min}$ with a resistance of $8 \mathrm{~cm} \mathrm{H}_{2} \mathrm{O}$ ) $\cdot$ liter $^{-1} \mathrm{~s}$ and a dead space of less than $2.5 \mathrm{ml}(10)$. The averaged GG and DIA EMGs, esophageal pressure, airflow, and mask pressure were recorded on a 6-channel Gould chart recorder.

A mean $( \pm \mathrm{SD})$ of $13 \pm 4$ end-expiratory occlusions were performed per infant with $8 \pm 3$ performed in active sleep and 5 \pm 3 in quiet sleep. After the nasal mask was manually occluded at end expiration, the occlusion was held until at least three occluded efforts were recorded or for a maximum of $10 \mathrm{~s}$. Occlusions were separated by at least 60 -s intervals and those resulting in behavioral arousal were not analyzed. Occlusions

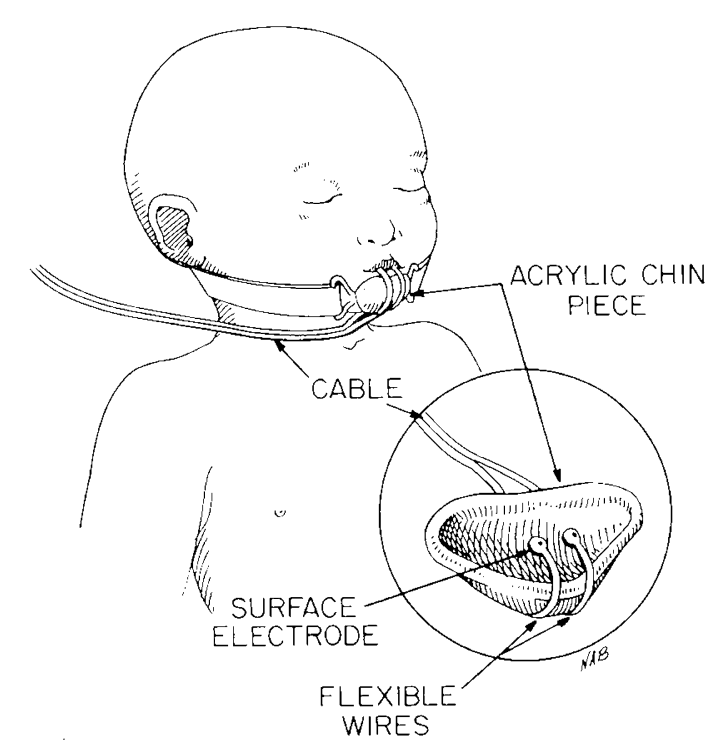

Fig. 1. Schematic illustration of the apparatus used to measure the genioglossus EMG via the sublingual electrodes used in the study. were only performed when oxygen saturation was at least $95 \%$ as measured by pulse oximetry (Nellcor-100, Nellcor Incorporated Haywood, CA). After $\geq 10$ min of sleep, behavioral criteria were utilized to assess sleep state with active sleep being associated with rapid eye movements, body movements, and an irregular respiratory pattern. Quiet sleep was characterized by a regular respiratory pattern and the absence of rapid eye and body movements with the exception of occasional startles. Each infant exhibited three min of active or quiet sleep before occlusions were performed. Data were only collected and analyzed when there was no subsequent change in sleep state.

Occlusions were analyzed with respect to: 1) the frequency of occurrence of phasic GG EMG activity associated with the first three occluded inspiratory efforts in active and quiet sleep;2) the timing of onset of the DIA and GG EMG during the occluded inspiratory efforts, in relation to the initial negative deflection of the esophageal pressure swing; 3 ) the prolongation of inspiratory time of the first occluded effort as compared to the breath preceding occlusion; 4) the magnitude of the esophageal pressure deflection of the first occluded inspiratory effort. A mean response to occlusion was calculated separately for each infant and the combined means were then subjected to statistical analysis. Ti was measured from the onset to the peak of the esophageal pressure swing for both the unoccluded breath and the occluded inspiratory effort. The percent of inspiratory prolongation was calculated from the equation $\left[\left(\mathrm{Ti}_{\text {occl }}-\mathrm{Ti}_{\text {unoccl }) / \text { occl }}\right)\right] \times 100$ where $\mathrm{Ti}_{\text {occl }}$ represents the first occluded effort and $\mathrm{Ti}_{\text {unocel }}$ represents the preceding unoccluded breath.

Results were analyzed by authors E.B.G. an J.M.D., one of whom was unaware as to which group the infants belonged. Statistical tests were analysis of variance with repeated measures and the Newman-Keuls procedure, the Student's paired and unpaired $t$ test and Mann-Whitney $U$ test as appropriate. Results are presented as mean \pm SEM.

\section{RESULTS}

Sporadic bursts of phasic GG EMG were recorded during arousal, nonnutritive sucking, swallowing, and apnea. Spontaneously occurring phasic GG EMG associated with respiration was infrequent and not sustained in either group of infants. In contrast, occlusion usually elicited a brisk GG response. As the response of the GG to airway occlusion was comparable in both sleep states, the combined data are presented for each group of infants.

The frequency of phasic GG EMG differed between control and apneic infants during occlusion. A representative tracing from each group is shown in Figures $2 A$ and $B$. In the control group $42 \pm 5 \%$ of occlusions were accompanied by a phasic $G G$ EMG on the 1st occluded inspiratory effort. The frequency of this response increased to $74 \pm 5 \%(p<0.01)$ and $80 \pm 5 \%(p$ $<0.01)$ by the second and third occluded inspiratory efforts, respectively. In contrast, in the infants with apnea only $13 \pm 4 \%$ of occlusions were associated with a phasic GG EMG on the 1st occluded inspiratory effort which increased to $38 \pm 9 \%(p)<$ $0.01)$ and $52 \pm 9 \%(p<0.01)$ by the second and third occluded inspiratory efforts, respectively. As indicated in Figure 3, the percent of occlusions with a GG response was less in the apneic as compared to the control infants $(p<0.001)$. When the data were subjected to analysis of variance with repeated measures, using gestational and postconceptual age as separate covariates, the GG response still differed significantly (both $p<0.01$ ) between the two groups of infants.

The onset of the phasic GG and DIA EMGs during the occluded inspiratory efforts was compared to the onset of the esophageal pressure deflection for the first three occluded efforts exhibiting a GG EMG. A delay in onset of the GG EMG was typically observed during occluded inspiratory efforts. This delay decreased with each subsequent appearance of the GG EMG as demonstrated in Figure $2 A$. The control infants had a delay of 


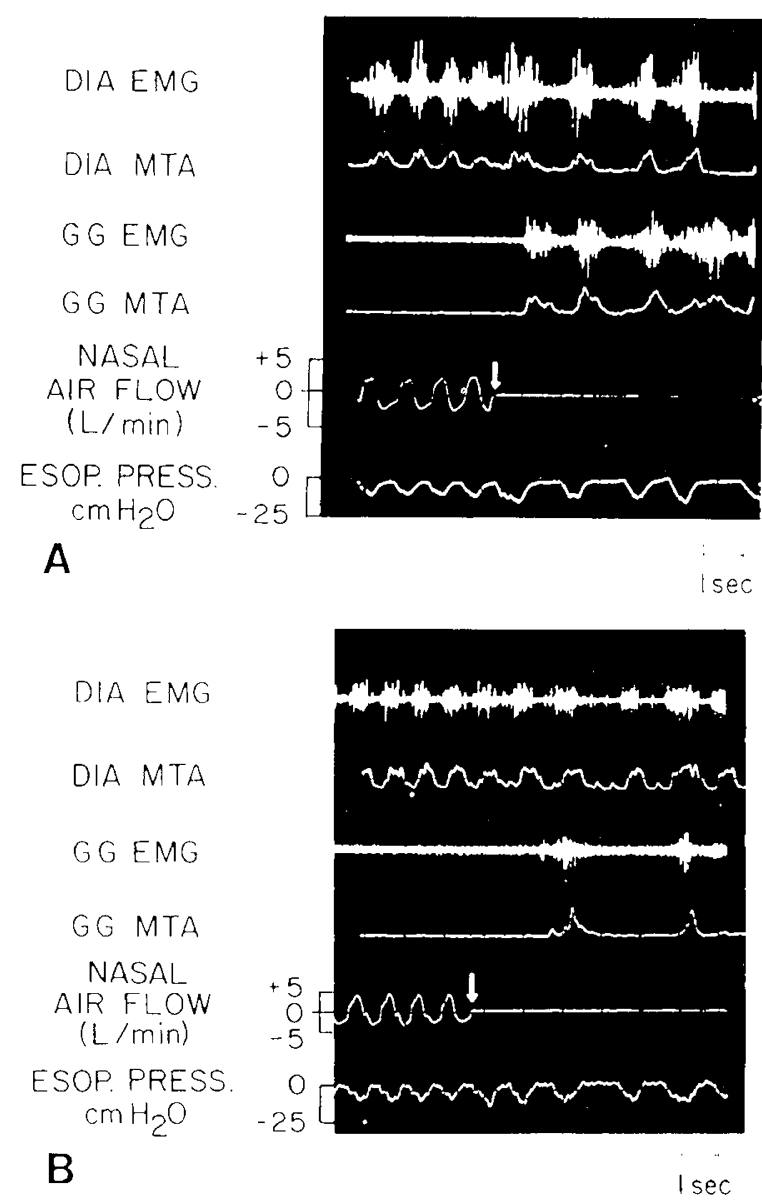

Jig. 2. 1. typical response of the (i $;$ FMe; in a control infant prior to and during occlusion. The arron depects the start of an end-expiators airway occlusion. Both raw and moving time averaged $(1 / 7 / 1)$ signals are presented for the (is $i$ and I)IA FMC is. Fach occluded inspiratory

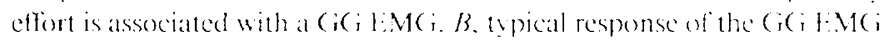

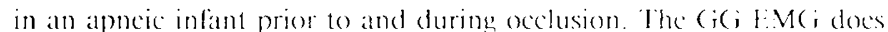
not appear until the thed oceluded inspiratory effort and reappears with the fifth occluded inspiratory effort.

$360 \pm 50.170 \pm 40$. and $50 \pm 60 \mathrm{~ms}$ on the first three appearances of the (i i EMC $i$ during occlusion. respectively. The infants with apnea had a comparable decrease of the delay from $350 \pm 20.10$ $110 \pm 60$ and 9()$\pm 5() \mathrm{ms}$ during occlusion. $\Lambda$ signiticant decrease in this delay occurred from the first to the second appearance of the $(;) ;: M(;$ in both groups of infants (Fig. 4). The I)IA I:M( $;$ was present with each breath or occluded inspiratory effort and preceded the onset of the esophageal pressure swing by $110 \pm 5$ ms in the control infants and $80 \pm 10$ in the apne infants for the first three occluded inspiratory efforts that were associated

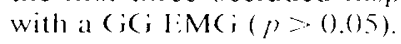

The magnitude of the esophageal pressure deflection of the first oceluded inspiratory effort was compared between the two groups. The control and apncic infants generated $-9 \pm 3$ and $-8 \pm 2 \mathrm{~cm} \mathrm{II},(0)$. respectively $(p=0.5)$ during occlusion.

Inspiratory time of the lirst oceluded effort was significantly prolonged as compared to the breath preceding the occlusion for both groups of infants (lig. 5). The percent prolongation of inspiratory time for the control infants $(35 \pm 8 \%)$ did not differ significantly from that in the apneic infants $(30 \pm 6 \%, p=0.5)$. The results were recalculated after omitting those occlusions

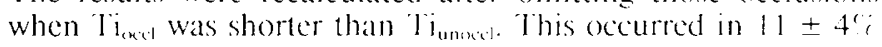
of the occlusions in the control infants and in $17 \pm 4 \%$ of the occlusions in the apneic infants. The two groups of infants still had comparable pereent prolongation of $1 \%,+1 \pm 5 \%$ in the controt and $40 \pm 5 \%$, in the apnecic group $(/)=(0.9)$.

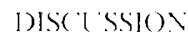

We did not ohserve sustained phasie inspiratory (is i FMC with unobstructed breathing in cither of our infant groups. Phasic

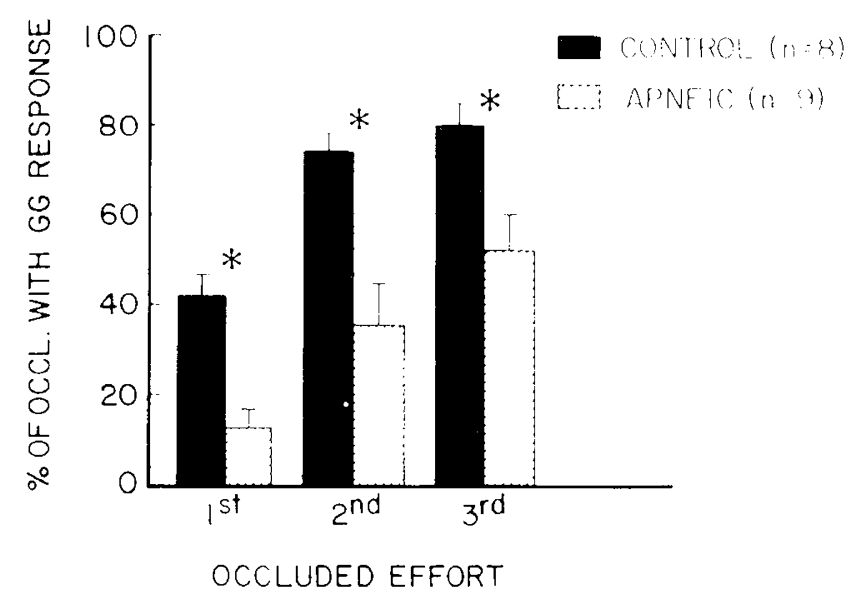

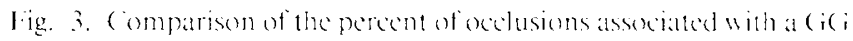
Me $;$ on the tirst. second. and third oceluded effert. Here was a significant difference hetween the response of control and alpucie intams $(* p<0(1).(0) 1)$.

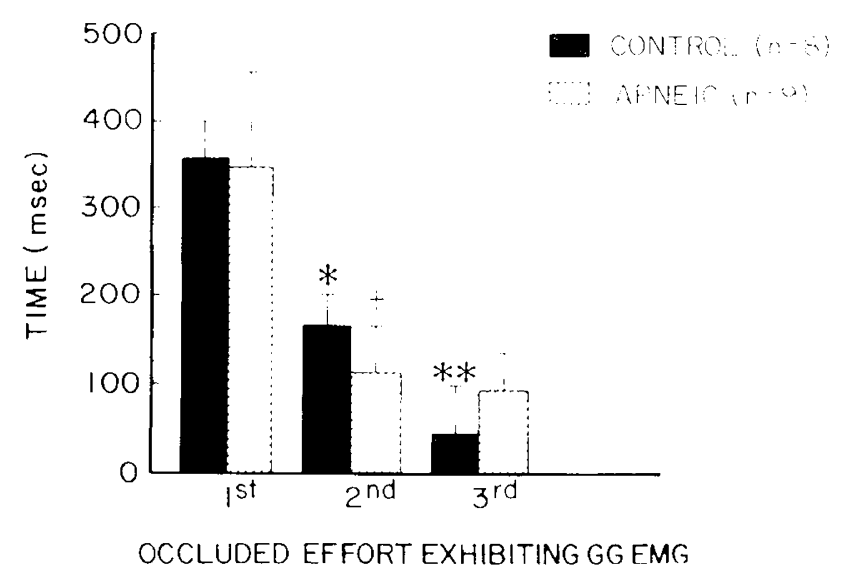

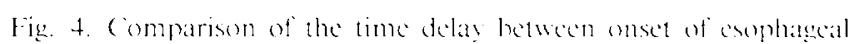

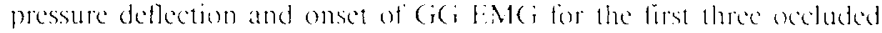

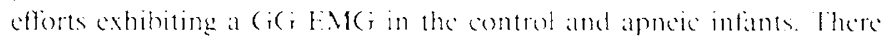
was a signiticant decrease in this delas when the second chlort was

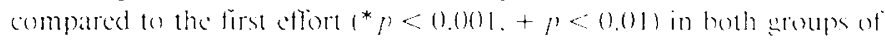

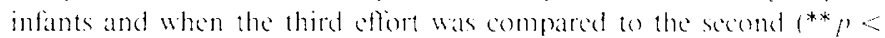
0.051 in the control group.

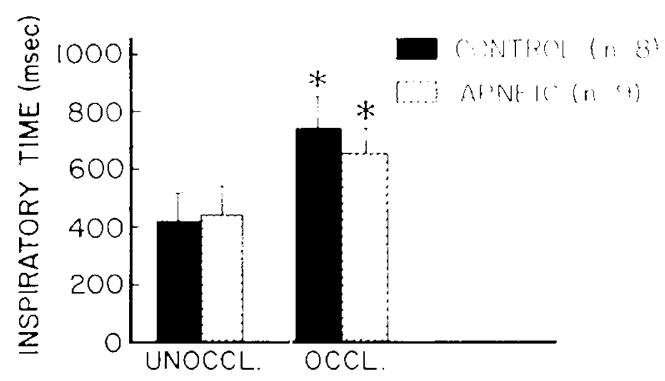

Fig. 5. Comparison of the inspiratory time bewwen the first oceluded inspiratory effort and the last unoceluded breath for the control and apneic infants $(* p<0.05)$. The prolongation of insplatory time dicl not differ between control and apnexic infints. 
activity occurring with inspiration has been recorded from the GG, using intramuscular electrodes in adults with and without sleep apnea syndrome $(1,6,11)$ although there is a paucity of data reporting the overall incidence of phasic GG activity during normal tidal breathing. Furthermore, it has been documented that sustained inspiratory activity of the GG during unobstructed breathing does not always occur in animals, children, or micrognathic or preterm infants $(2,5,12-14)$. The activity of the GG is augmented, however, during hypercarbia, hypoxia, and endexpiratory airway occlusion $(5-7,13,14)$. Our data in premature infants indicate that GG activity may be absent during unobstructed breathing but can be clearly elicited during occlusion.

We have shown that infants with apnea have less augmentation of their GG EMG during occlusion than nonapneic controls. Previous data suggest that lung inflation preferentially inhibits the activity of the upper airway muscles via a vagal reflex (HeringBreuer) arising from pulmonary stretch receptors (15-17). Thus, the absence of lung inflation during end-expiratory occlusion may produce an increase in the activity of the upper airway muscles due to the release of this inhibition $(2,13,17)$. Alteration in afferent activity from pressure or flow receptors in the upper airway may also contribute to augmentation of the GG during occlusion $(18,19)$. Esophageal pressure should reflect upper airway pressure provided the airway remains patent and this did not differ between the two groups of infants on the first occluded inspiratory effort. Therefore, it is unlikely that the decreased GG response in the apneic infants resulted from less negative pressure in the upper airway during occlusion.

The Hering-Breuer reflex is thought to contribute to the prolongation of the first inspiratory effort in response to airway occlusion in infants $(20,21)$. The intercostal phrenic reflex may also influence inspiratory time as chest wall distortion during inspiration may shorten inspiratory time (22), especially during occlusion. Therefore, in response to occlusion two opposing mechanoreceptor reflexes, the Hering-Breuer and the intercostal phrenic reflex, may be affecting inspiratory duration in premature infants. When the occlusions associated with shortening of inspiratory time were removed from the analysis, the two groups of infants still had comparable inspiratory prolongation. Thus, our data indicate that the strength of the Hering-Breuer reflex does not appear to differ between apneic and nonapneic infants. This is in contrast to the findings of Gerhardt and Bancalari (23) who observed significant differences in the prolongation of inspiration following occlusion in apneic versus nonapneic premature infants. Our study had $80 \%$ power to detect a difference of $30 \%$ or more in the percent prolongation of inspiratory time between the two groups with group sizes of nine and eight, at $p$ $<0.05$. The infants reported by Gerhardt and Bancalari (23) had a mean postnatal age of 8 days while our infants had a mean postnatal age of 3 and $4 \mathrm{wk}$ for the control and apneic infants, respectively. This difference in postnatal age may have contributed to the contrasting findings between these two studies. Our data indicate that the decreased response of the GG during occlusion in the apneic infants cannot be explained by a difference in mechanoreceptor influence on the activity of the upper airway muscles.

We speculate that the decreased GG response during occlusion in the infants with apnea may result from an overall decrease in efferent output to the muscles of the upper airway. Gerhardt and Bancalari (24) demonstrated decreased $\mathrm{CO}_{2}$ sensitivity in apneic infants when compared to controls at a mean gestation of $30 \mathrm{wk}$, and speculated that the infants with apnea had an abnormality in the central control of breathing. In addition, studies evaluating brainstem evoked potentials have also found prolonged brainstem conduction times in apneic infants suggestive of immature brainstem function (25). Contrary to adult and animal data, when the phasic GG EMG appeared during occlusion it did not precede the DIA EMG or the onset of the corresponding inspiratory effort (5-7). Both groups of infants, however, had an earlier appearance of the GG EMG with each subsequent firing of the
GG during occlusion. It is possible that the sensitivity of the surface electrodes did not allow us to detect the true onset of EMG activity. However, the electrodes were placed directly over the origin of insertion of the GG, and this position was the optimal placement of recording EMG activity with needle electrodes as described by Sauerland and Harper (11). In addition, Doble et al. (18) reported good correlation between sublingual surface electrodes and intramuscular electrodes in adults. The use of surface electrodes, however, may have limited our ability to detect very low level phasic activity. It is likely that the upper airway muscles are inhibited during unobstructed breathing (13, $15,16)$. The delay in activation of the GG during occlusion may be secondary to release of this inhibition or stimulation that occurs after the onset of the occluded inspiratory effort. The progressive decrease in this delay with each subsequent appearance of the GG EMG during occlusion is probably the effect of increased chemoreceptor and/or mechanoreceptor stimulation.

Although the two groups were of comparable postconceptional age, and the difference in the response to occlusion between the two groups was still present when the data were subjected to an analysis of variance with gestational age used as a covariate, the relative immaturity of the apneic infants at the time of birth may have still influenced our results. Seven of the nine infants in the apneic group were being treated with theophylline. Theophylline has been shown to be a central respiratory stimulant (26). Therefore this is unlikely to have contributed to the decreased GG response to occlusion in the apneic infants.

We conclude that the GG is usually not active during unobstructed breathing in premature infants. During end-expiratory airway occlusion, there appears to be release of the inhibitory control with resultant augmentation of the GG. However, premature infants with mixed and obstructive apnea have less augmentation of the GG during induced occlusion than control infants, possibly due to an overall decrease in central output to the muscles of the upper airway. Our findings suggest that infants with mixed or obstructive apnea are less apt to recruit their upper airway dilating muscles in response to spontaneous obstruction, thereby prolonging the airway obstruction and its consequences.

\section{REFERENCES}

1. Remmers JE. deGroot WJ, Sauerland EK, Anch AM 1978 Pathogenesis of upper airway occlusion during sleep. J Appl Physiol 44:931-938

2. Roberts JL, Reed WR. Mathew OP, Thach BT 1986 Control of respiratory activity of the genioglossus muscle in micrognathic infants. J Appl Physiol 61:1523-1533

3. Milner AD, Boon AW. Saunders RA. Hopkin IE 1980 Upper airways obstruction and apnoea in preterm babies. Arch Dis Child 55:22-25

4. Mathew OP, Roberts JL, Thach BT 1982 Pharyngeal airway obstruction in preterm infants during mixed and obstructive apnea. J Pediatr 100:964-968

5. Haxhiu MA, van Lunteren E, Mitra J, Cherniack NS 1984 Responses to chemical stimulation of upper airway muscles and diaphragm in awake cats. J Appl Physiol 56:397-403

6. Onal E, Lopata M, O'Connor TD 1981 Diaphragmatic and genioglossal electromyogram responses to $\mathrm{CO}_{2}$ rebreathing in humans. $\mathrm{J}$ Appl Physiol 50:1052-1055

7. Patrick GB, Strohl KP, Rubin SB, Altose MD 1982 Upper airway and diaphragm muscle responses to chemical stimulation and loading. J Appl Physiol 53:1133-1137

8. Doble EA, Leiter JC, Knuth SL, Daubenspeck JA, Bartlett D Jr 1985 A noninvasive intraoral electromyographic electrode for genioglossus muscle. J Appl Physiol 58:1378-1382

9. Evanich MJ, Lopata M, Lourenco RV 1978 Analytic methods for the study of electrical activity in respiratory nerves and muscles. Chest 70 (suppl 1):158S$162 \mathrm{~S}$

10. Anderson JV Jr, Martin RJ, Lough MD, Martinez A 1982 An improved nasal mask pneumotachometer for measuring ventilation in neonates. $J$ App Physiol 53:1307-1309

11. Sauerland EK, Harper RM 1976 The human tongue during sleep: clectromyographic activity of the genioglossus muscle. Exp Neurol 51:160-170

12. Jeffries B, Brouillette RT, Hunt CE 1984 Electromyographic study of some accessory muscles of respiration in children with obstructive sleep apnea Am Rev Respir Dis 129:696-702

13. Carlo WA, Miller MJ, Martin RJ 1985 Differential response of respiratory muscles to airway occlusion in infants. J Appl Physiol 59:847-852

14. Parisi RA, Neubauer JA, Frank MM, Edelman NH, Santiago TV 1987 Correlation between genioglossus and diaphragmatic responses to hypercapnia during sleep. Am Rev Respir Dis 135:378-382 
15. van lunteren IE, Strohl KP. Parker I)M. Bruce LX. Van de (irath HB. (herniack VS 1984 Phasic volume-related feedbatek on upper airwas muscle activity. J Appt Physiol $5(0: 7.30)-7.36$

16. Kuna $S E$ 1986 Inhibition of inspiratory upper airway motoneuton activity by phasic volume feedback. 1 Appl Physiol 60:137.3. 1.379)

17. Brouillette RT, Thach BI [980 (ontrol of genioglossus musche inspirators activity I Appl Phosiol 49:80)1-808

18. Mathew (OP, Abu-Osba YK Thach BI 1982 (conioglossus muscle responses 10 upper airway pressure changes: afferent pathways. J Appl Physiol 52:445. 450

19. Mathew ()P. Abu-()sba YK. Thach B I 1982 Influence of upper airwaly pressure

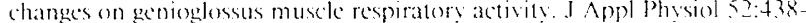
4.44

20. Olinsky A. Bryan MH. Bryan A( 1974 Influence of lung inflation on respira-

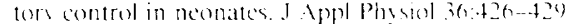

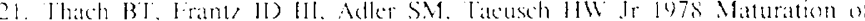
reflexes influencing inspiraturs duration in human intants I Appl phasiol $45: 2(1) 32: 211$

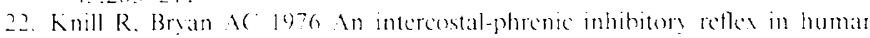
mewhorn infants. 1 Appl Phusiol $40,3: 3-3.50$

23. (ierhardt 1. Bancalan 1: 1984 Apnea of prematurits: Il. Respimator tedlexes Pediatrice 7.603..606

24. (iefhardt I. Bancalart 1: 1984 apnea of prematurity: 1. 1 ung lunction and regulation of heratheng. Pediatrice $74: 58-62$

25. Henderson-Smant D). Pottigrew A ( brainstem neural function in preterm infints. 2 lingl J Med $308: 35: 35$ ?

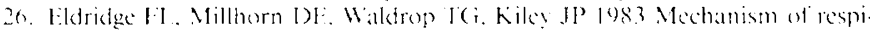
rators effects of methelxanthines. Respir Phisiof 53:230 261 